\title{
AutoTune Report
}

This report displays the results of the MRM development carried out as part of an autotune.

Calibration method : C:\MassLynx\DEFAULT.PRO\AcquDB\calibration.cal

MSMS Tune method : C:\MassLynx\Cinnarizinne.pro\ACQUDB\CNN.ipr

Daughter search data file : C:\MassLynx\Cinnarizinne.PRO\data\Product.raw

Date: Generated on Wed 21 Mar 2018 at 13:44

Cone Optimize Chromatogram for m/z 369.18

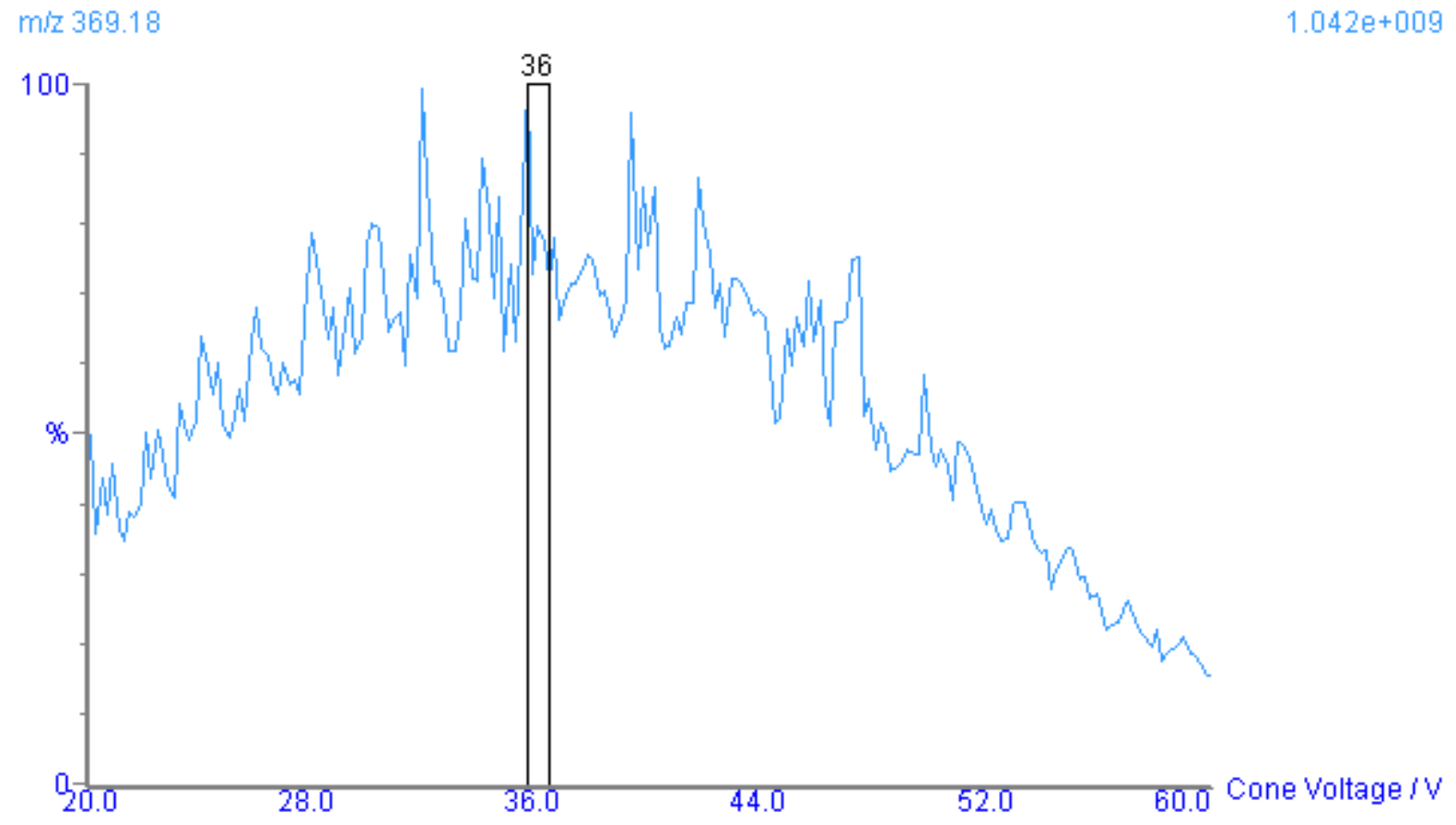

Cone Optimize Spectrum for m/z 369.18 


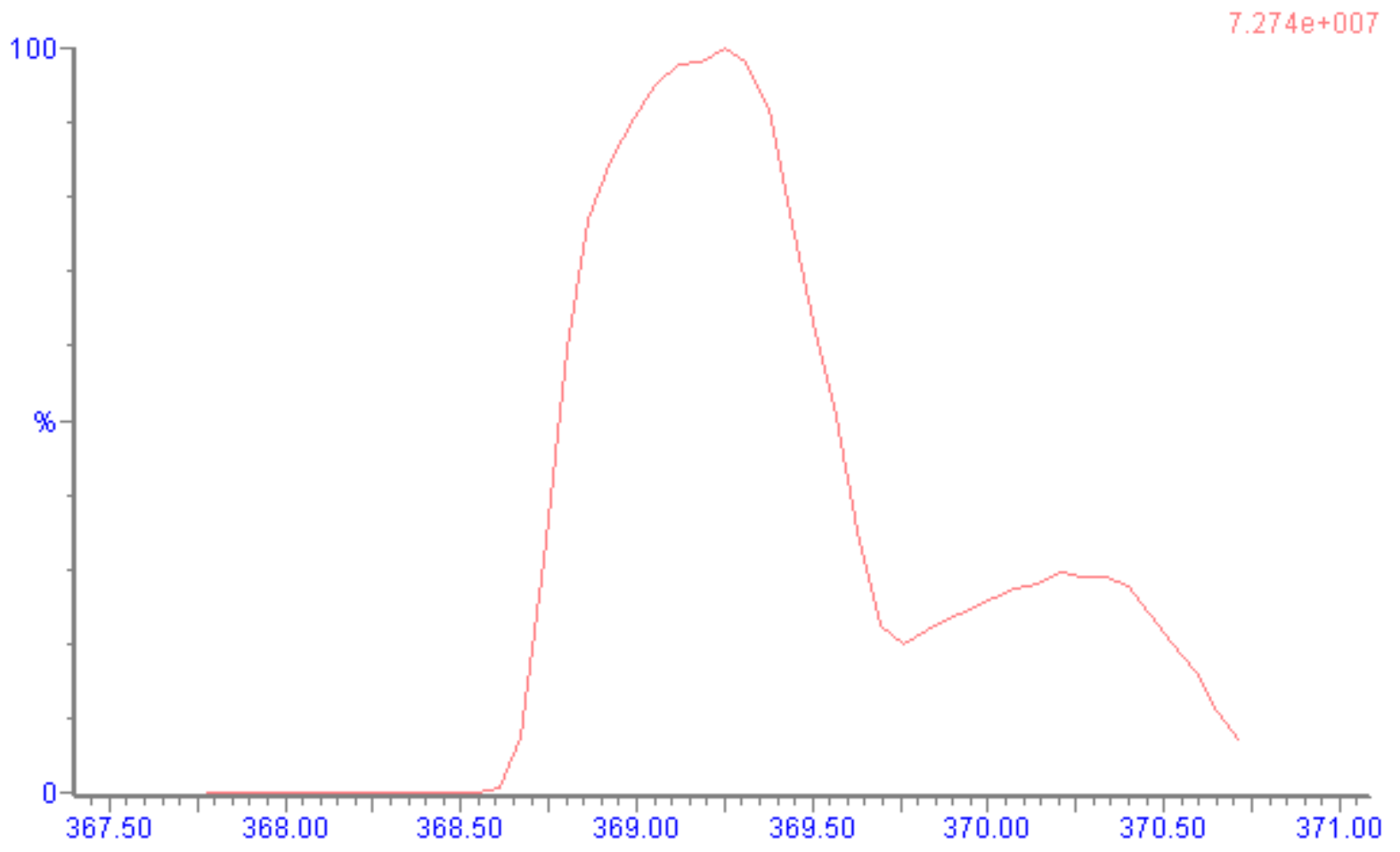

The MRM experiment developed: C: \MassLynx\Cinnarizinne.pro\ACQUDB \CNN Method.exp

\begin{tabular}{||l|l|l|l|}
\hline Precursor Mass & Product Mass & Cone Voltage & Collision Energy \\
\hline 369.18 & 152.0 & 36.00 & 48.00 \\
\hline \hline 369.18 & 167.0 & 36.00 & 16.00 \\
\hline 369.18 & 201.1 & 36.00 & 12.00 \\
\hline
\end{tabular}

Collision Energy Optimize Chromatogram for m/z 369.18 -> 152.0 


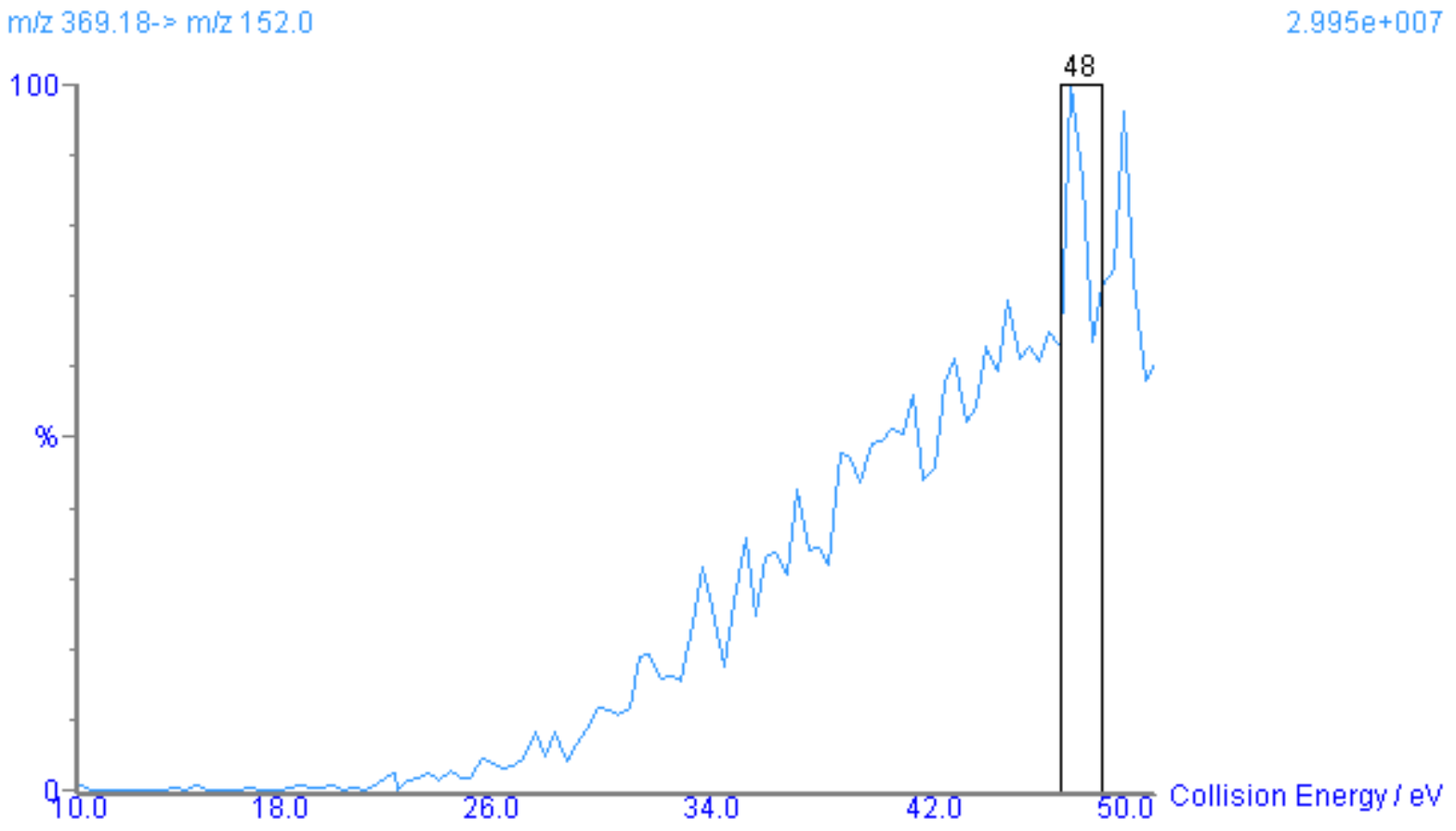

Collision Energy Optimize Spectrum for m/z 369.18 -> 152.0

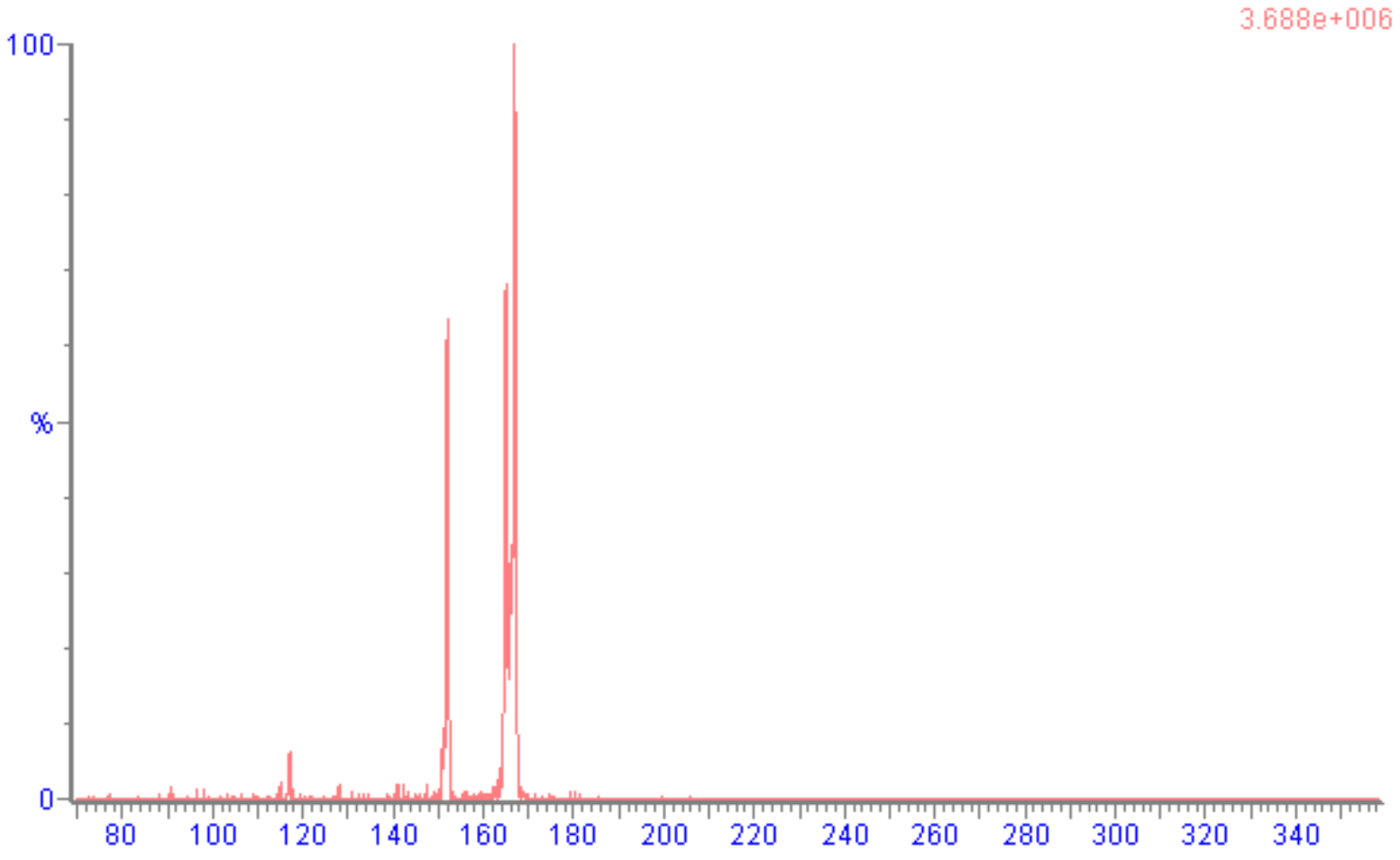

Collision Energy Optimize Chromatogram for m/z 369.18 -> 167.0 


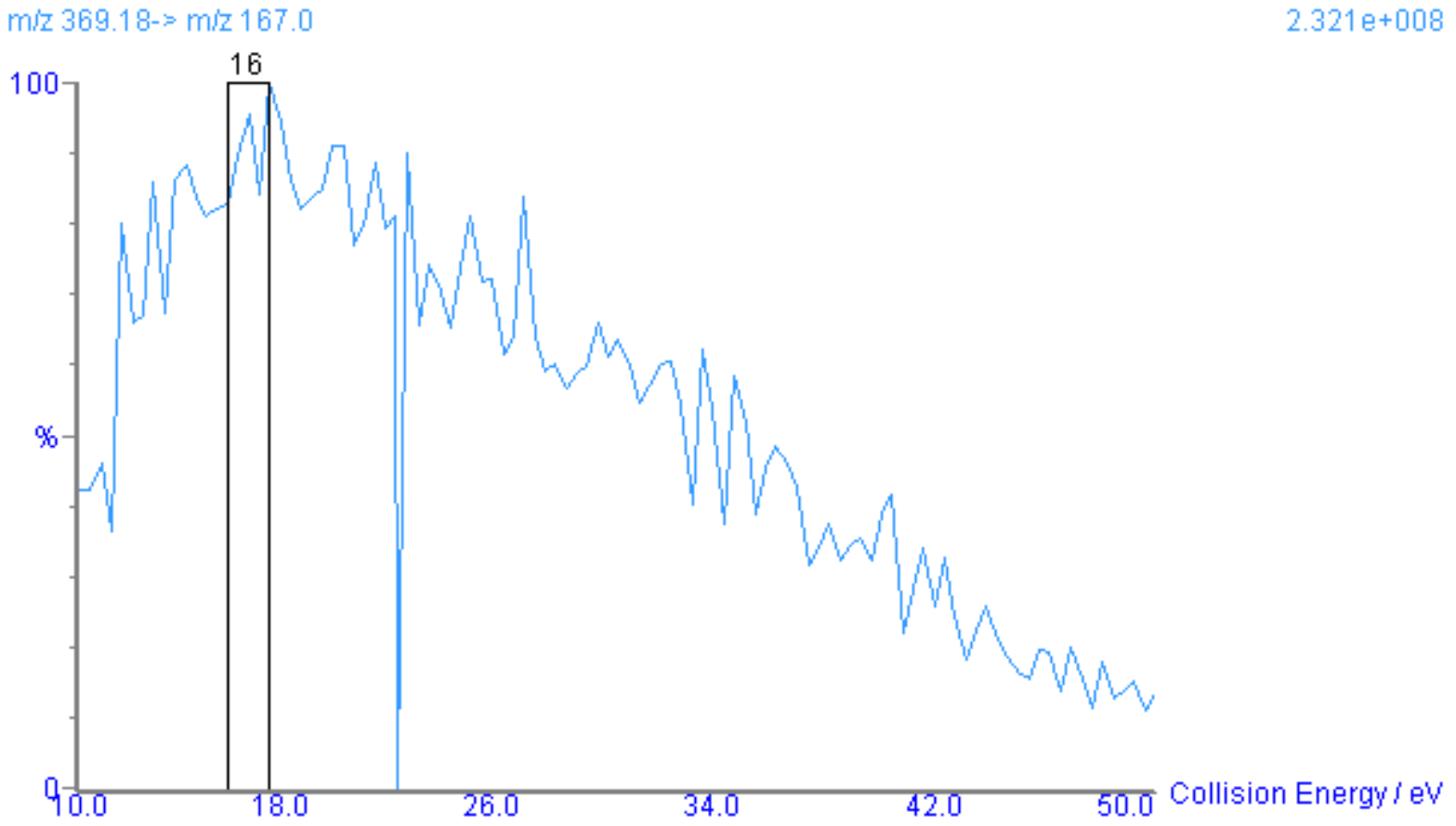

Collision Energy Optimize Spectrum for m/z 369.18 -> 167.0

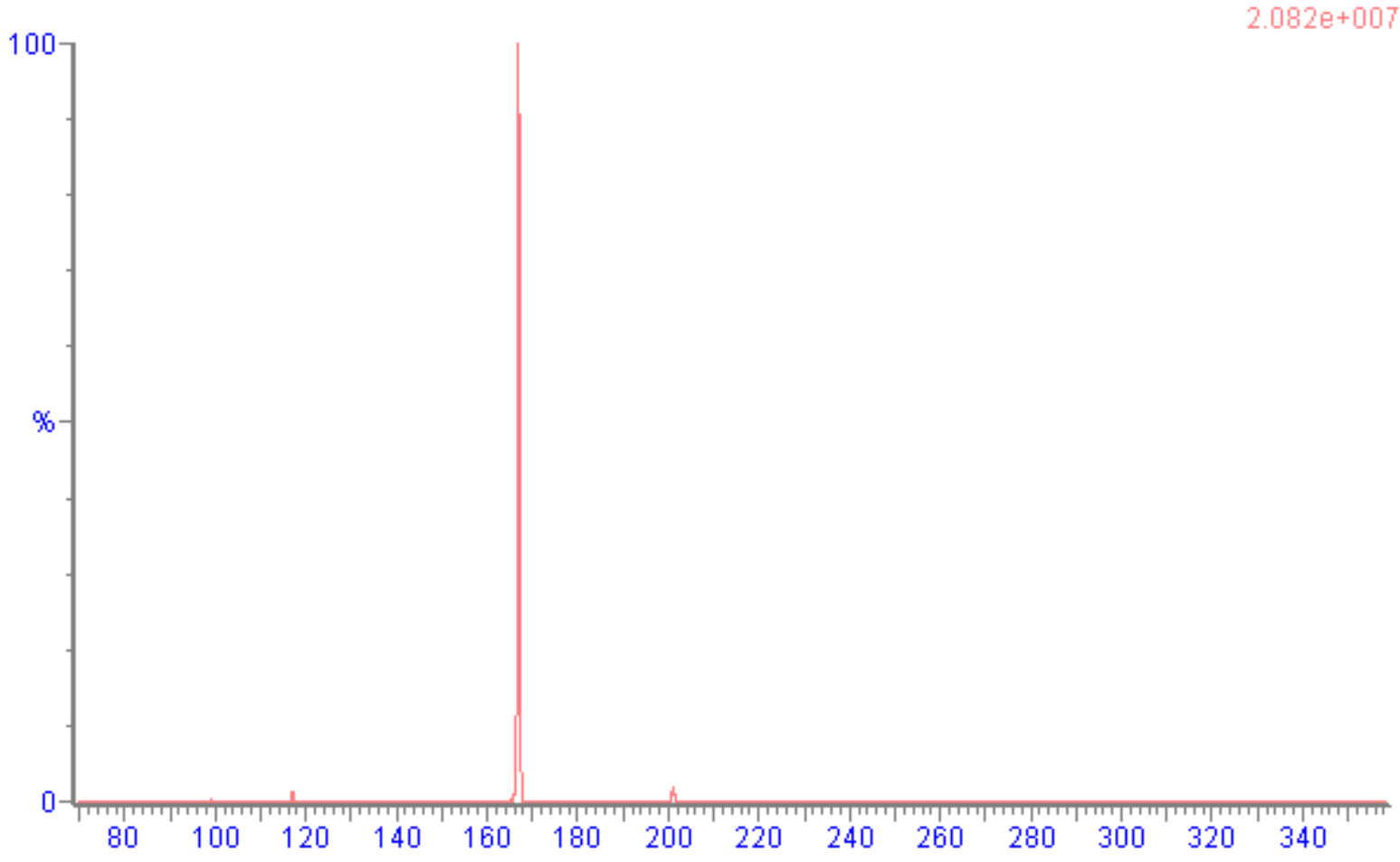

Collision Energy Optimize Chromatogram for m/z 369.18 -> 201.1 


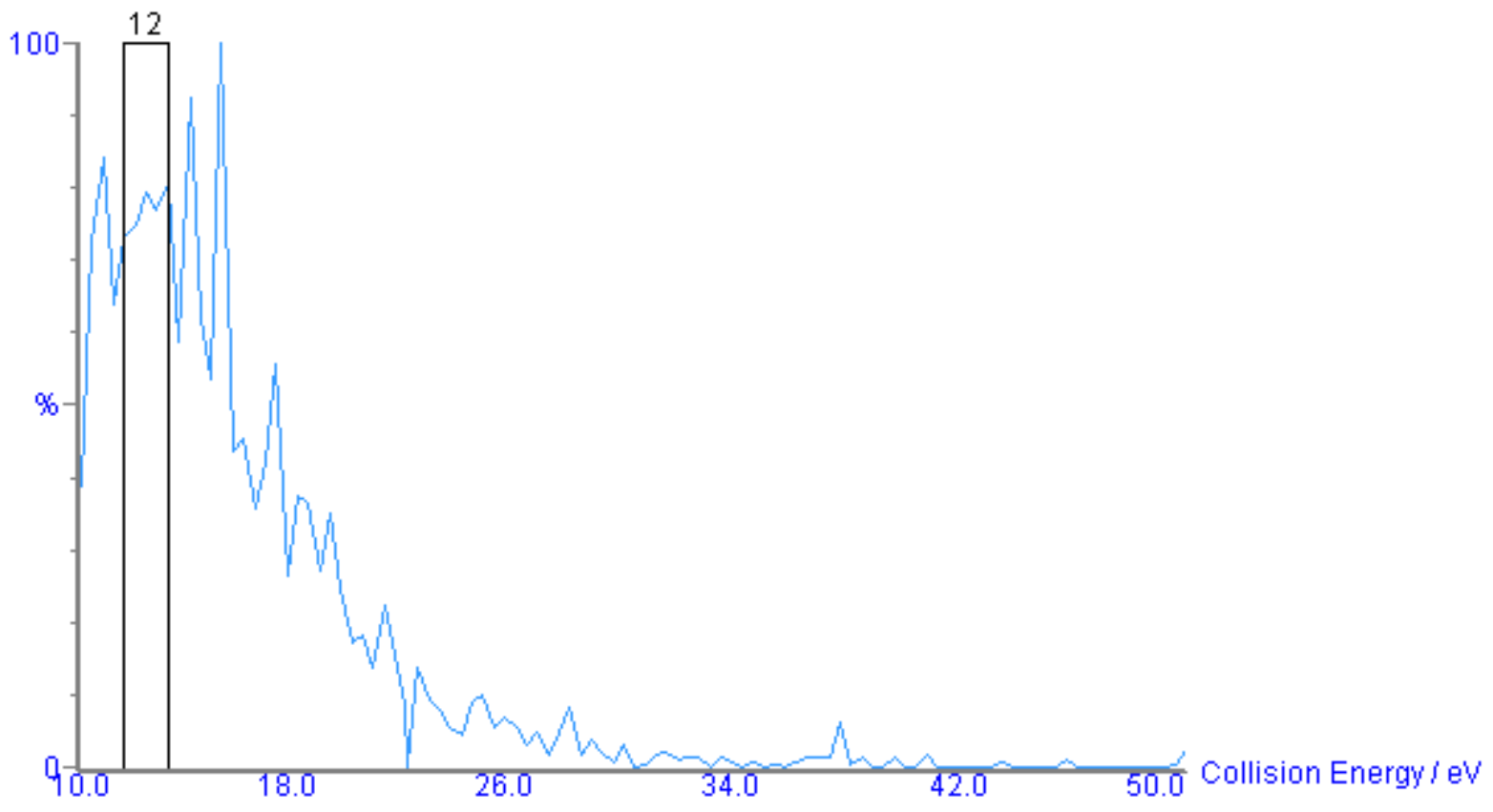

Collision Energy Optimize Spectrum for m/z 369.18 -> 201.1

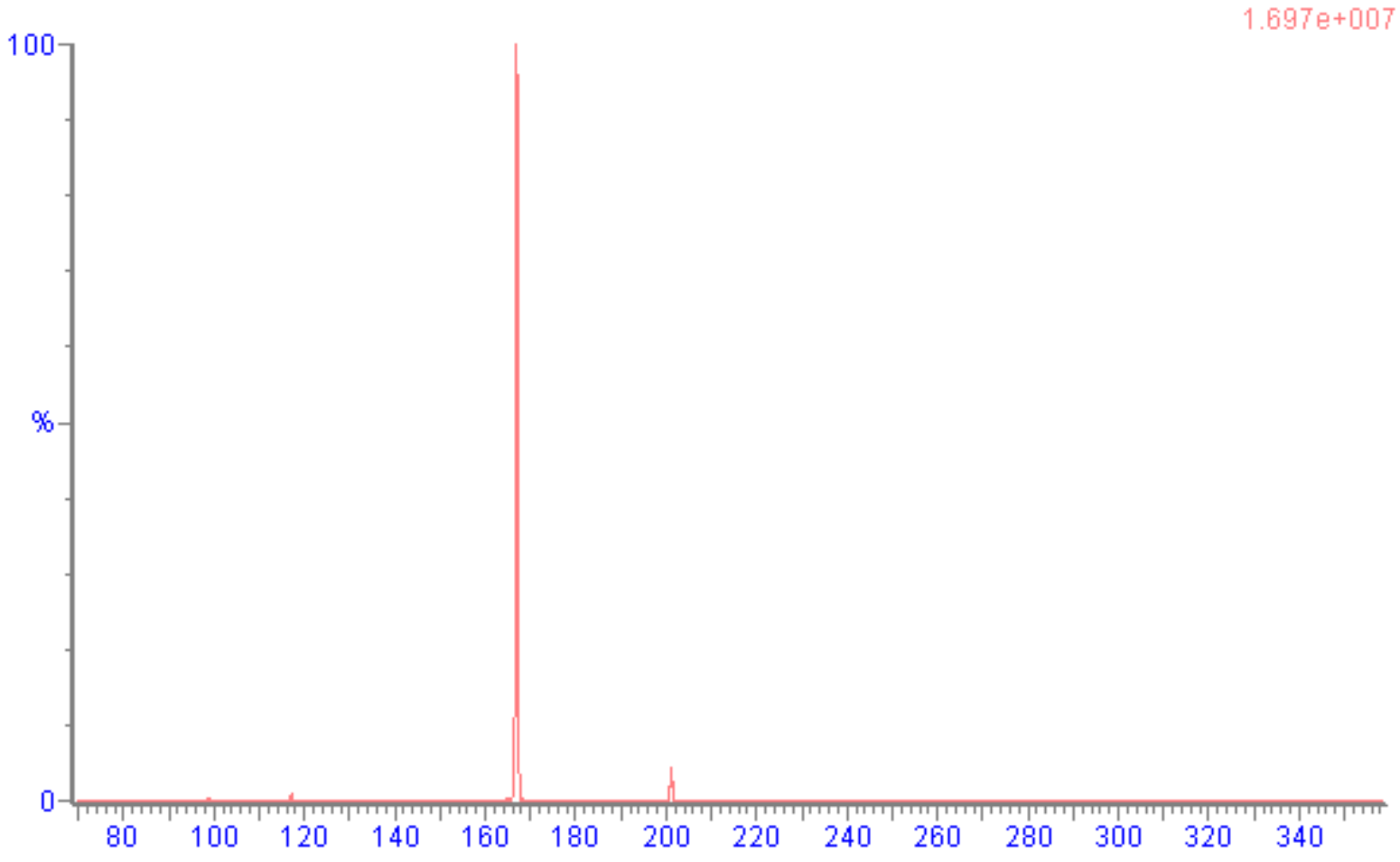

